\title{
Estudo de prevalência de defeitos congênitos no Vale do Paraíba Paulista
} Prevalence study of birth defects in Vale do Paraíba, São Paulo, Brazil

Cilene Otaviano Pinto ${ }^{1}$, Luiz Fernando C. Nascimento ${ }^{2}$

\section{RESUMO}

Objetivo: Estimar a prevalência de anomalias congênitas no Vale do Paraíba Paulista em 2002 e 2003.

Métodos: Estudo transversal com base em dados constantes na Declaração de Nascido Vivo (DNV), cujas informações estavam no portal da Secretaria da Saúde do Estado de São Paulo. As malformações foram descritas de acordo com o capítulo XVII do Código Internacional de Doenças (CID) 10 , referente a variáveis maternas e do recém-nascido. A variável desfecho (dependente) foi a presença de anomalia congênita; as demais informações das mães e do recém-nascido constantes na DNV (variáveis independentes) foram analisadas para estimar as associações entre elas e a variável desfecho. Utilizou-se o programa Epi-Info 6.04d para análise estatística e o teste do qui-quadrado, do qui-quadrado de tendência linear e o teste $t$ de Student.

Resultados: Foram analisados 41.838 dados com informações constantes nas DNVs, sendo identificados $618(1,5 \%)$ sem preenchimento do campo correspondente à anomalia congênita e $317(0,76 \%)$ nascidos com anomalia congênita. Houve associação positiva de anomalias congênitas com menor duração da gestação, maior número de filhos mortos, tipo de parto, baixo peso ao nascer e menor escore de Apgar. Os sistemas mais afetados foram o osteomuscular e o nervoso.

Conclusões: A prevalência de malformações foi menor que a encontrada em outros estudos, possivelmente por subregistro de informação.

Palavras-chave: recém-nascido; anormalidades; declaração de nascimento; prevalência.

\section{ABSTRACT}

Objective: Estimate the prevalence of birth defects in the Vale do Paraíba Paulista, São Paulo - Brazil, during the years of 2002 and 2003.

Methods: Cross-sectional study based on Birth Certificates available in the São Paulo Health Secretary site. The abnormalities were described according to chapter XVII of the International Classification of Diseases and Related Health Problems (ICD) 10 for variables related to mothers and infants born alive at birth. The dependent variable was the presence of abnormalities. The independent variables were analyzed to estimate the association between congenital anomalies and maternal and newborn data registered in the Birth Certificates. Qui-square test, linear trend qui-square and Student $t$ test were performed by the Epi-Info 6.04d statistical program.

Results: We analyzed 41,838 records, but $628(1.5 \%)$ birth certificates did not present the specific field fulfilled and $317(0.76 \%)$ neonates presented birth defects. There was a positive association between congenital anomalies and premature delivery, stillbirths, type of delivery, low birth weight and low Apgar score. The osteomuscular and nervous systems were most frequently affected.

Conclusions: The prevalence of malformations observed in the present study was lower than those described in other studies, possibly due to under notification.

Key-words: infant, newborn; abnormalities; birth certificates; prevalence.

\footnotetext{
${ }^{1}$ Acadêmica do Departamento de Medicina da Universidade de Taubaté (Unitau)

${ }^{2}$ Professor assistente doutor do Departamento de Medicina da Unitau e doutor em Saúde Pública pela Universidade de São Paulo

Endereço para correspondência:

Luiz Fernando C. Nascimento

Rua Durval Rocha, 500 - Vila Paraíba
}

CEP 12515-710 - Guaratinguetá/SP

E-mail: Ifcn@unitau.br

Fonte financiadora: Fundação de Amparo à Pesquisa do Estado de São Paulo (Fapesp), com bolsa de Iniciação Científica, processo n. ${ }^{\circ}$ 05/54598-8

Recebido em: 2/3/2007

Aprovado em: 10/7/2007 


\section{Introdução}

Os dados relativos à prevalência de malformações congênitas no Brasil são escassos; o mesmo ocorre em todo o Estado de São Paulo. Há um estudo do período compreendido entre os anos de 1985 e 1995 , que avaliou o impacto ambiental decorrente da extração de carvão e sua repercussão na saúde reprodutiva, no Rio Grande do Sul ${ }^{(1)}$. Essa pesquisa mostrou 45 casos de malformações congênitas no período (4,34 por 1.000 nascidos vivos), sendo semelhante aos dados do Estudo Colaborativo Latino-Americano de Malformações Congênitas (ECLAMC) ${ }^{(1)}$. Também no Rio Grande do Sul, foram identificadas prevalências de algumas malformações associadas à idade materna inferiores às idades observadas nos dados do ECLAMC ${ }^{(2)}$.

Alguns estudos mostram que a presença de malformações atinge aproximadamente $3 \%$ dos nascidos vivos ${ }^{(3)}$. Quanto a defeitos específicos como os do fechamento do tubo neural, a prevalência é de 4,16 por 1.000 nascidos vivos ${ }^{(4)}$. Recentemente, utilizando a Declaração de Nascido Vivo (DNV), foi possível estimar a prevalência de malformações de 0,88\% no ano de 2001, em uma cidade paulista ${ }^{(5)}$.

A DNV é um instrumento básico do Sistema de Informações sobre Nascidos Vivos (Sinasc), de responsabilidade do Ministério da Saúde, implantada no Brasil a partir de $1990^{(6)}$. Ela possui informações, entre outras, sobre o local de ocorrência do nascimento, dados maternos, da gestação, tipo de parto e algumas características do recém-nascido. Habitualmente, utiliza-se esse instrumento no estudo da influência de variáveis maternas sobre o peso ao nascer ${ }^{(7)}$. Uma das vias dessa declaração é entregue ao cartório de registro civil, outra permanece anexada ao prontuário hospitalar do recém-nascido e, por fim, outra é enviada à Secretaria da Saúde do Estado.

Dados referentes à prevalência de malformações congênitas podem ser obtidos facilmente por meio da DNV. Desde 1999, o Ministério da Saúde introduziu um campo específico (campo 34) para malformações congênitas e/ou anomalias cromossômicas e seu código correspondente no Capítulo XVII da Classificação Internacional das Doenças (CID) $10^{(8,9)}$.

O objetivo do presente estudo foi estimar a prevalência de malformações congênitas no Vale do Paraíba Paulista, em 2002 e 2003, utilizando os dados de DNV, que estão consolidados no portal eletrônico da Secretaria da Saúde do Estado de São Paulo.

\section{Métodos}

Estudo do tipo transversal baseado em dados secundários da DNV, obtidos da Secretaria da Saúde do Estado de São Paulo e relativos ao Vale do Paraíba Paulista, a respeito de mães residentes nesta região, com filhos nascidos nos anos de 2002 e 2003 . Foram analisados dois anos para aumentar a chance de encontrar malformações congênitas, pois alguns municípios poderiam apresentar poucos partos.

Os bancos de dados utilizados foram obtidos a partir do portal eletrônico http://www.saude.sp.gov.br/informacoes_saude/sistemas/html/informacoes_sistemas_base_dados. html. Os dados que compõem este banco fornecem informações constantes na DNV, mas estas não foram acessadas diretamente. Foram considerados os municípios de residência das mães e não os municípios de ocorrência dos partos.

O estudo abrangeu inicialmente os 39 municípios pertencentes às Diretorias Regionais de Saúde (DIR) XXI e XXIV, que correspondem a, respectivamente, São José dos Campos e Taubaté, atingindo uma população aproximada de 2 milhões de habitantes. Foram excluídos cinco municípios que possuíam mais de $8 \%$ de não preenchimento do campo 34 da DNV, referente às anomalias congênitas. A população dos municípios excluídos corresponde a cerca de 250 mil habitantes. Foram consideradas as áreas urbanas e as rurais.

O Vale do Paraíba Paulista desenvolve basicamente atividades relacionadas à indústria, agropecuária e mineração. A região de São José dos Campos foi a que apresentou o maior salto quantitativo do Estado de São Paulo entre 1996 e 2001 (de 6,5\% da indústria estadual para 11\%), graças à refinaria de petróleo Henrique Lage e outras indústrias voltadas para a exportação (aeronáutica e metalúrgica, principalmente), segundo pesquisa da Atividade Econômica Paulista de 2001 realizada pela Fundação Sistema Estadual de Análise de Dado (Seade) ${ }^{(10)}$.

A variável de desfecho (dependente) foi a presença e a identificação da anomalia congênita. As variáveis independentes estudadas para possíveis associações com as anomalias congênitas foram: idade materna, escolaridade materna, número de filhos tidos em gestações anteriores - nascidos vivos e nascidos mortos, duração da gestação, tipo de parto, número de consultas de pré-natal, gênero do recém-nascido, escore de Apgar no quinto minuto e peso ao nascer.

A idade materna foi categorizada em até 19 anos, de 20 a 34 anos e mais de 35 anos. A escolaridade materna foi classificada em ensino fundamental (até oito anos de esco- 
laridade), médio (de nove a 11 anos) e superior (12 anos ou mais). O número de filhos nascidos vivos foi categorizado em 0,1 a 2, e 3 ou mais. O número de filhos nascidos mortos foi categorizado em 0,1 e 2, ou mais.

A duração da gestação foi categorizada em menor que 32 semanas, de 32 a 36 semanas e 37 semanas ou mais. $\mathrm{O}$ tipo de parto foi vaginal ou cesáreo. O número de consultas de pré-natal foi categorizado em até seis consultas e sete ou mais consultas. O índice de Apgar no quinto minuto foi categorizado segundo nota 0 a 3 , nota 4 a 7 e nota 8 a 10. O peso ao nascer foi classificado em normal, quando o peso ao nascimento era igual ou superior a $2.500 \mathrm{~g}$, e em baixo peso, quando inferior a $2.500 \mathrm{~g}$.

Os dados foram analisados pelo programa Epi-info versão $6.04 \mathrm{~d}$. Para avaliar a associação, foi utilizada a estatística do qui-quadrado e, para as variáveis ordinais, o qui-quadrado de tendência linear. As médias de variáveis quantitativas como peso do recém-nascido, escore de Apgar de cinco minutos, idade materna, número de filhos vivos e filhos mortos em gestações anteriores foram comparadas pelo teste $t$ de Student. O nível de significância estatística foi definido em $5 \%$. Foram criados intervalos de confiança de $95 \%$ para os valores de malformações, utilizando-se da distribuição de Poisson, visto se tratar de evento raro.

Nas tabelas, os casos com informação ignorada não foram considerados, havendo, portanto, variação no número total de fichas analisadas. Por se tratarem de dados disponíveis na rede e por não serem identificados, não são feridos aspectos éticos.

\section{Resultados}

No período estudado de dois anos, foram analisadas 41.838 DNVs de recém-nascidos de mães residentes nos municípios considerados. Neste total, não se incluíram cinco municípios com mais de $8 \%$ de não preenchimento do campo 34 da DNV. Estes cinco municípios perfizeram 9.394 declarações excluídas do estudo, representando $18,3 \%$ do total de partos $(\mathrm{N}=51.232)$. Foram incluídos no estudo, 21.520 recém-nascidos do gênero masculino $(51,4 \%), 20.315$ do gênero feminino $(48,6 \%)$ e três com gênero indeterminado.

O peso médio ao nascer foi de 3.132 5528 g. A idade média das mães foi $25 \pm 6$ anos. O Apgar médio de quinto minuto foi de $9 \pm 1$. O número de filhos vivos e mortos anteriores foi de $1,2 \pm 1,4$ e $0,2 \pm 0,5$, respectivamente.
O não preenchimento do campo correspondente às anomalias congênitas ocorreu em 628 (1,5\%) DNVs. O gênero dos recém-nascidos foi o campo melhor preenchido, não havendo ausência de preenchimento. Ao contrário, o campo relativo à presença de filhos mortos em gestações anteriores não foi preenchido em $24 \%$ das declarações.

A Tabela 1 mostra a distribuição dos recém-nascidos e os casos e porcentagens dos neonatos com anomalias congênitas, segundo as variáveis do estudo. Observa-se, ainda na Tabela 1, a porcentagem de campos que não foram preenchidos, bem como os dados das variáveis e os respectivos níveis descritivos. Houve associação, com significância estatística, entre as anomalias congênitas e as variáveis: número de filhos mortos, duração da gestação, tipo de parto, Apgar de quinto minuto e baixo peso ao nascer. Por outro lado, não foi possível identificar associação entre anomalias congênitas e as variáveis idade e escolaridade maternas, assim como com a ordem de nascimento, número de consultas no pré-natal e gênero do recém-nascido (Tabela 1 ).

Nas fichas estudadas, a ocorrência de anomalias congênitas foi de 317 casos, representando 7,57 casos de anomalias congênitas/1.000 nascidos vivos. As anomalias mais freqüentes foram: 150 (47,3\% do total das malformações) casos de anomalias do sistema osteomuscular, representadas por pé torto congênito, sindactilia e polidactilia; $42(13,2 \%)$ de malformações de sistema nervoso, representados por mielocele e mielomeningocele; e 37 casos $(11,6 \%)$ de fenda labial e fenda palatina. Houve 14 casos de Síndrome de Down, que é uma anomalia cromossômica incluída para apontar a atenção à identificação de malformações. Estes dados, assim como os intervalos de confiança de $95 \%$ estão mostrados na Tabela 2.

\section{Discussão}

Trata-se do primeiro estudo epidemiológico realizado em todos os municípios do Vale do Paraíba Paulista sobre a prevalência de anomalias congênitas com dados constantes na DNV. Só foram consideradas informações de nascidos vivos que se encontravam nos bancos de dados obtidos da Secretaria da Saúde do Estado de São Paulo.

Dos 39 municípios da região, foram incluídos 34; estes possuíam menos de $8 \%$ de não preenchimento da DNV no campo relativo às malformações. Houve municípios com alta porcentagem de fichas não preenchidas, atingindo até $69 \%$ de não preenchimento. 
Tabela 1 - Distribuição de malformações congênitas no Vale do Paraíba (n=317), nos anos de 2002 e 2003, segundo as variáveis associadas

\begin{tabular}{|c|c|c|c|c|}
\hline & $\mathrm{n}^{\circ}$ de indivíduos (\%) & $\mathrm{n}^{\circ}$ de malfo & rmações (\%)" & $p$ \\
\hline Idade materna $10-19$ anos & $7.686(18,4)$ & 50 & $(0,6)$ & 0,11 \\
\hline Idade materna $20-35$ anos & $30.768(73,5)$ & 233 & $(0,7)$ & 0,06 \\
\hline Idade materna $>35$ anos & $3.363(8,0)$ & 34 & $(1,0)$ & \\
\hline Ausência de anotação & $21 \quad(0,1)$ & & - & \\
\hline Escolaridade materna até 8 anos & $14.921(35,7)$ & 117 & $(0,8)$ & 0,60 \\
\hline Escolaridade materna 9-11 anos & $20.557(49,1)$ & 149 & $(0,7)$ & 0,87 \\
\hline Escolaridade materna $>11$ anos & $5.908(14,1)$ & 50 & $(0,8)$ & \\
\hline Ausência de anotação & $452(1,1)$ & 1 & $(0,2)$ & \\
\hline Ausência de filhos nascidos vivos & $12.993(31,0)$ & 96 & $(0,7)$ & 0,63 \\
\hline Um ou 2 filhos nascidos vivos & $19.018(45,5)$ & 154 & $(0,8)$ & 0,98 \\
\hline Três ou mais filhos nascidos vivos & $5.036(12,0)$ & 35 & $(0,7)$ & \\
\hline Ausência de anotação & $4.791(11,5)$ & 32 & $(0,6)$ & \\
\hline Ausência de filhos nascidos mortos & $27.180(65,0)$ & 196 & $(0,7)$ & 0,012 \\
\hline Um filho nascido morto & $3.725(8,9)$ & 44 & $(1,2)$ & 0,022 \\
\hline Dois ou mais filhos nascidos mortos & $837(2,0)$ & 7 & $(0,8)$ & \\
\hline Ausência de anotação & $10.096(24,1)$ & 70 & $(0,7)$ & \\
\hline Duração da gestação <32 semanas & $411(1,0)$ & 0 & $(0)$ & $<0,001$ \\
\hline Duração da gestação 32 a 36 semanas & $2.072(4,9)$ & 47 & $(2,3)$ & $<0,001$ \\
\hline Duração da gestação >37 semanas & $38.806(92,8)$ & 266 & $(0,7)$ & \\
\hline Ausência de anotação & $549(1,3)$ & 4 & $(0,7)$ & \\
\hline Parto normal & $19.250(46,0)$ & 121 & $(0,6)$ & 0,006 \\
\hline Parto cesáreo & $22.456(53,7)$ & 195 & $(0,9)$ & \\
\hline Ausência de anotação & $132(0,3)$ & 1 & $(0,7)$ & \\
\hline Até 6 consultas de pré-natal & $13.946(33,3)$ & 116 & $(0,8)$ & 0,25 \\
\hline Mais de 7 consultas de pré-natal & $27.037(64,7)$ & 195 & $(0,7)$ & \\
\hline Ausência de anotação & $855 \quad(2,0)$ & 1 & $(0,1)$ & \\
\hline Gênero masculino & $21.520(51,4)$ & 174 & $(0,8)$ & 0,22 \\
\hline Gênero feminino & $20.315(48,6)$ & 142 & $(0,7)$ & \\
\hline Indefinido & $3(0,0)$ & & $(33,3)$ & \\
\hline Ausência de anotação & - & & - & \\
\hline Apgar 5 minutos: 0 a 3 & $152(0,4)$ & 25 & $(16,4)$ & $<0,001$ \\
\hline Apgar 5 minutos: 4 a 7 & $852 \quad(2,0)$ & 29 & $(3,4)$ & $<0,001$ \\
\hline Apgar 5 minutos: 8 a 10 & $40.184(96,0)$ & 257 & $(0,6)$ & \\
\hline Ausência de anotação & $650 \quad(1,6)$ & 6 & $(0,9)$ & \\
\hline Baixo peso $(<2.500 \mathrm{~g})$ & $3.877 \quad(9,2)$ & 82 & $(2,1)$ & $<0,001$ \\
\hline Peso normal $(\geq 2.500 \mathrm{~g})$ & $37.961(90,8)$ & 235 & $(0,6)$ & \\
\hline Ausência de anotação & - & & & \\
\hline
\end{tabular}


Dos municípios excluídos, somente um apresentava população ao redor de 140 mil habitantes; os demais eram de pequeno porte, com população ao redor de 20 mil habitantes. Assim, a exclusão dessas informações, que estavam concentradas em apenas cinco municípios, possivelmente não influenciou o resultado final; além disso, eram municípios com as mesmas características daqueles que permaneceram na análise. Como a cobertura do Sinasc foi de $100 \%$ na região estudada, existe a confiabilidade nos dados.

Em relação a outros estudos, podem ser citados os da Colômbia, onde foi encontrada uma prevalência de 4,4\% de anomalias congênitas em recém-nascidos e 7,8\% em natimortos $^{(11)}$; e, no Chile ${ }^{(12)}$, onde houve maior prevalência de malformações em conceptos de mães adolescentes. Em outro estudo chileno, no período de 1995 a 1999, dentre 12.735 nascimentos, registraram-se 980 nascidos vivos com malformações $(7,74 \%)$ e 15 malformações entre os natimortos ${ }^{(13)}$. Recentemente, Costa $e a^{(14)}$ identificaram prevalência de $1,7 \%$ de anomalias congênitas na cidade do Rio de Janeiro. Os autores encontraram os mesmos fatores associados a anomalias congênitas observados no presente estudo, isto é, baixo peso, prematuridade e menores escores de Apgar. No estudo do ECLAMC, com duração de 30 anos, a prevalência de defeitos do tubo neural apresentou tendência secular de aumento e foi maior em mães com filhos mortos e com filhos vivos de peso inferior a $1.500 \mathrm{~g}$ e em mães menores de 19 anos de idade ${ }^{(15)}$.
No presente estudo, entre os nascidos vivos estudados, a ocorrência de anomalias congênitas nos anos de 2002 e 2003 foi de 317 casos $(0,76 \%)$, valores estes acima dos encontrados no Rio Grande do Sul, onde foram analisados somente dez tipos de defeitos congênitos e sua ocorrência foi de 45 casos $(0,43 \%)$ em 10.391 nascidos vivos ${ }^{(1)}$.

No entanto, os valores encontrados no presente estudo estão abaixo daqueles relatados em um hospital de Bogotá ${ }^{(1)}$ e ligeiramente inferiores ao observado no Vale do Paraíba Paulista, com valores de 0,88 casos/100 NV em São José dos Campos em 2001 ${ }^{(5)}$. Tais diferenças possivelmente se devem à forma de obter as informações, podendo ocorrer vieses de amostragem e aferição, o que explicaria os valores discrepantes. Por outro lado, alguns defeitos podem se manifestar mais tardiamente, após a alta hospitalar do recém-nascido, o que subestimaria a sua ocorrência.

As anomalias congênitas mais freqüentemente encontradas foram as do sistema osteomuscular, sistema nervoso e a fenda labial e palatina. Esses dados se assemelham aos de Caracas e aos encontrados no Rio de Janeiro, nos quais as malformações de sistema osteomuscular, cardíacas e neurológicas foram as mais freqüentes ${ }^{(15,16)}$.

Dentre os achados do presente estudo, houve uma associação estatística entre a presença de anomalias congênitas e as variáveis: menor duração da gestação, menor escore de Apgar no quinto minuto, baixo peso ao nascimento e tipo de parto. Em relação à associação entre baixo peso e presença de malformaç̃oes, os dados coincidem com os encontrados em Bogotá, Colômbia ${ }^{(11)}$ e também com os de São José dos Campos $^{(5)}$.

Tabela 2 - Distribuição de malformações congênitas no Vale do Paraíba Paulista ( $n=317$ ), nos anos de 2002 e 2003, de acordo com capítulo XVII da CID-10

\begin{tabular}{lcc}
\hline Localização das malformações & Malformações (\%) & IC95\%\$ $^{\S}$ \\
\hline Sistema osteomuscular & $150(47,3)$ & $127-174$ \\
Sistema nervoso & $42(13,2)$ & $30-55$ \\
Fenda labial e fenda palatina & $37(11,7)$ & $26-49$ \\
Aparelho circulatório & $11(3,5)$ & $5-18$ \\
Olho, ouvido, face e pescoço & $10(3,2)$ & $4-17$ \\
Outras malformações do aparelho digestivo & $10(3,2)$ & $4-17$ \\
Órgãos genitais & $6(1,9)$ & $2-11$ \\
Aparelho respiratório & $5(1,6)$ & $1-10$ \\
Aparelho urinário & $1(0,3)$ & $0-3$ \\
Outras malformações congênitas & $29(9,1)$ & $19-40$ \\
Não classificadas em outra parte* & $16(5,0)$ & $9-24$ \\
\hline
\end{tabular}

"Incluem malformações congênitas múltiplas, não classificadas em outra parte (exclui síndromes com malformações congênitas que acometem múltiplos sistemas), outras malformações congênitas especificadas e malformações congênitas não especificadas; "Síndrome de Down e Síndrome de Turner; sobtido pela Distribuição de Poisson, bicaudal, em valores absolutos 
Quanto ao baixo peso, fatores genéticos ou ambientais podem ser responsáveis pela presença de malformações congênitas e estas estariam implicadas na gênese do baixo peso, explicando, assim, a associação entre a presença de malformações congênitas e o baixo peso ao nascer.

Quanto à duração da gestação, houve associação entre malformações e a menor duração da gestação (pré-termo) tanto no teste de associação do qui-quadrado, como do qui-quadrado de tendência linear. Não se sabe se as malformações podem ter sido o fator causal da menor duração de gestação. É possível que as malformações comprometam o desenvolvimento da criança intra-útero e desencadeiem o parto prematuro. Além disso, quando o concepto é portador de defeito congênito, seria mais freqüente a interrupção eletiva da gestação antes do termo, na tentativa de proporcionar ao recém-nascido um tratamento mais eficaz.

As malformações estiveram associadas ao escore de Apgar baixo no quinto minuto. Também as proporções de malformações congênitas diminuíram com o aumento da nota de Apgar. Não foi possível obter uma explicação para este fato.

Houve associação positiva entre parto cesáreo e presença de recém-nascidos com malformações congênitas; talvez este fato se deva ao diagnóstico de malformações intra-útero e a cesárea tivesse sido a opção médica, corroborando com a hipótese levantada para explicar a prematuridade.

Por outro lado, a porcentagem de malformações encontrada neste estudo foi inferior à da literatura mundial, a qual relata valores ao redor de $3 \%$ de prevalência de anomalias congênitas ${ }^{(3)}$, sendo explicada, possivelmente, pela forma de coleta da informação. Outros estudos que utilizem informações da DNV poderão confirmar ou não a prevalência encontrada no presente estudo.

A discrepância entre os resultados observados no Vale do Paraíba Paulista e na literatura mundial pode ser devida a diferenças sazonais, ambientais e regionais ou devido à ausência de observação de alguns casos de malformações congênitas, como, por exemplo, as cardiopatias congênitas, que podem ter sido diagnosticadas dias após o nascimento e depois da alta hospitalar. É importante lembrar que a forma de coleta de informações a respeito das anomalias congênitas também pode explicar estas diferenças.

Apesar de não encontrarmos dados a respeito de doenças maternas na DNV, estes não podem ser ignorados para explicar a presença de malformações congênitas. Muitas doenças maternas crônicas estão associadas a problemas fetais e neonatais. Há maior chance de malformações em filhos de mães com asma brônquica, diabetes melito, hipertensão e hipotiroi- dismo, comparada à chance de anomalias em filhos de mães saudáveis ${ }^{(17)}$. Pesquisa chilena demonstrou que mães diabéticas têm freqüência dez vezes maior de malformações congênitas e cinco vezes maior de aborto espontâneo ${ }^{(18)}$. Do mesmo modo, a DNV não contempla o passado médico da gestante.

No que concerne às diferenças regionais encontradas, pode ser destacado o papel da poluição ambiental em alguns municípios do Vale do Paraíba Paulista, como São José dos Campos. Estudo desenvolvido na Califórnia encontrou associação entre defeitos congênitos cardíacos (defeitos septais, defeitos valvulares e das artérias pulmonar e aorta) e exposição aumentada a monóxido de carbono e ozônio no segundo mês de gestação ${ }^{(19)}$. O estudo mostrou que o mecanismo de ação dos poluentes atmosféricos ocorreria por meio de eventos hipóxicos e hemodinâmicos, estresse oxidativo e toxicidade a certas células durante o desenvolvimento. É sabido que o monóxido de carbono e o ozônio podem produzir malformações no esqueleto de animais.

O uso dos dados constantes nas DNVs para estimar a prevalência de malformações congênitas pode ser mais confiável quando há poucos campos não preenchidos, sendo que, neste estudo, apenas 628 declarações mostravam o campo correspondente às anomalias congênitas não preenchidos (1,5\% do total). Na presente pesquisa, cinco municípios forma excluídos por contarem com mais de $8 \%$ de ausência de preenchimento de dados, talvez pelo não treinamento de pessoal responsável ou não percepção da importância do correto preenchimento. A alta porcentagem de preenchimento da DNV se deve, provavelmente, ao fato destas serem preenchidas no berçário, por profissionais da área de saúde. Isto permitiu obter dados fidedignos sobre a prevalência real de anomalias congênitas destas cidades pela possível redução do número de erros de preenchimento.

Além da falha do preenchimento da DNV, que ocorreu em alguns municípios, outra possível limitação do estudo é o fato de algumas malformações congênitas só se manifestarem após a alta hospitalar da mãe e do nascido vivo. Este viés poderia ser minimizado confrontando-se os dados de internações posteriores ao nascimento com as malformações congênitas observadas no preenchimento da DNV, para avaliar a ausência de informação. Seria interessante também que trabalhos futuros possam comparar a tendência secular de malformações congênitas do Vale do Paraíba Paulista com dados de outros municípios e Estados.

Conclui-se que as informações contidas na DNV, pelo fato desta ser preenchida por profissionais da área da 
saúde, permitem estudos de prevalência de malformações congênitas. No entanto, há ainda necessidade de um reforço no treinamento de médicos e profissionais de saúde que atuam em berçários, no sentido de melhor entenderem o papel da DNV, preenchendo todos os seus campos. A inclusão de outras informações relevantes na DNV, como história de doenças maternas na gestação, poderá mostrar outros fatores associados à gênese das malformações congênitas.

\section{Agradecimentos}

Cilene Otaviano Pinto agradece à Fundação de Amparo à Pesquisa do Estado de São Paulo (Fapesp), pelo apoio financeiro.

\section{Referências bibliográficas}

1. Leite JC, Schüler-Faccini L. Defeitos congênitos em uma região de mineração de carvão. Rev Saude Publica 2001;35:136-41.

2. Oliveira LM, Stein N, Sanseverino MT, Vargas VM, Fachel JMC, Schüler L. Reproductives outcomes in an area adjacent to a petrochemical plant in Southern Brazil. Rev Saude Publica 2002;36:81-7.

3. Monteleone-Neto R, Castilla EC, Lopez-Camelo JS. Reconhecimento do efeito teratogênico sobre o homem. In: Rabello-Gay MN, Rodrigues MALR, MonteleoneNeto R, editores. Mutagênese, teratogênese e carcinogênese: métodos e critérios de avaliação. Ribeirão Preto: Sociedade Brasileira de Genética; 1988. p. 197-271.

4. Aguiar MJ, Campos AS, Aguiar RA, Lana AM, Magalhães RL, Babeto LT. Defeitos do fechamento do tubo neural e fatores associados em recém-nascidos vivos e natimortos. J Pediatr (Rio J) 2003;79:129-34.

5. Nascimento LF, Pinto CO, Proença FP, Gotlieb SL. Prevalência de anomalias congênitas em São José dos Campos, SP, em 2001. Rev Paul Pediatr 2006;24:47-51.

6. Carniel EF, Antonio MA, Mota MR, Morcillo AM, Zanolli ML. A "Declaração de Nascido Vivo" como orientadora de ações de saúde em nível local. Rev Bras Saude Mater Infant 2003;3:165-74.

7. Nascimento LF, Gotlieb SL. Fatores de risco para o baixo peso ao nascer, com base em informações da Declaração de Nascido Vivo em Guaratinguetá, SP, no ano de 1998. Inf Epidemiol SUS 2001;10:113-20.

8. OMS. Organização Mundial da Saúde. CID-10: classificação estatística internacional de doenças e problemas relacionados à saúde. 10̊ revisão. São Paulo: Universidade de São Paulo; 1998.

9. Ministério da Saúde: Fundação Nacional de Saúde, Centro Nacional de Epidemiologia. Coordenação de Informação e Análise da Situação de Saúde. Sistema de informação sobre nascidos vivos: manual de instruções para o preenchimento da Declaração de Nascido Vivo. $3^{a}$ ed. Brasília: Centro de Documentação do Ministério da Saúde; 1999. Disponível em: http://www. saude.rio.rj.gov.br/saude/pubsms/media/dn_manual_preenchimento.pdf
10. São Paulo; Fundação SEADE. Pesquisa da Atividade Econômica Paulista de 2001. [citado em 12 de março de 2005].Disponível em: http://www.seade. gov.produtos/paes/pdfs/analiseregional.pdf

11. Garcia H, Salguero GA, Moreno J, Arteaga C, Giraldo A. Frecuencia de anomalías congénitas en el Instituto Materno Infantil de Bogotá. Biomédica (Bogotá) 2003;23:161-72.

12. Pardo RA, Nazer J, Cifuentes L. Prevalencia al nacimiento de malformaciones congénitas y de menor peso de nacimiento en hijos de madres adolescentes. Rev Med Chil 2003;131:1165-72.

13. Nazer J, Aravena T, Cifuentes L. Malformaciones congénitas en Chile: un problema emergente (período 1995-1999). Rev Med Chil 2001;129:895-904.

14. Costa CM, Gama SG, Leal Mdo C. Congenital malformations in Rio de Janeiro, Brazil: prevalence and associated factors. Cad Saude Publica 2006;22:2423-31.

15. Nazer J, Lopez-Camelo J, Castilla EE. ECLAMC: Estudio de 30 años de vigilancia epidemiológica de defectos de tubo neural en Chile y en Latinoamérica. Rev Med Chil 2001;129:531-9.

16. Arreaza E, Molina N, Peñuela H, Arcia O. Estudio transversal de Is malformaciones congénitas em recién nacidos de la Maternidad Concepción Palácios: Caracas: abril-julio de 1989. Arch Venez Pueric Pediatr 1992; 55:173-9.

17. Ordóñez MP, Nazer J, Áquila A, Cifuentes L. Malformaciones congénitas y patología crónica de la madre: estudio ECLAMC 1971-1999. Rev Med Chil 2003;131:404-11.

18. Nazer J, Ramirez FR. Malformaciones congénitas en los hijos de madres diabéticas. Rev Med Chil 2000;128:1045-52.

19. Ritz B, YU F, Fruin S, Chapa G, Shaw GM, Harris JA. Ambient air pollution and risk of birth defects in Southern California. Am J Epidemiol 2002;155:17-25. 